\title{
Body-image: Crossing science and art
}

\author{
Olga Pombo, Catarina Nabais, Marco Pina and Silvia Di Marco \\ Centre for Philosophy of Science, Faculty of Science, University of Lisbon \\ Campo Grande, C4, Room 4.3.24 \\ 1749-016 Lisbon, Portugal \\ opombo@fc.ul.pt, ccnabais@fc.ul.pt,marco.pina32@gmail.com, sdmarco@fc.ul.pt
}

\section{INTRODUCTION}

We live today under the empire of image. After oral and written words, image acquires an unprecedented relevance. It dramatically determines our life, both our way of seeing the world and of performing our individual and collective existence. Our aim is to present a video installation - Body-image - followed by a discussion where, taking images of the body as a case-study, questions related to the actual epistemological value of image will be discussed.

Body-image was created in 2011 by the Centre for Philosophy of Science of the University of Lisbon in the context of a research project entitled "Image in Science and Art". It shows images of the body produced by Science and Art since the $19^{\text {th }}$ century up until today. It is structured in three moments Body-landscape, Body-fragment, and Bodyalgorithm - accompanying the most crucial technical and technological transformations occurred in that period. The first is marked by drawing; the second by photography and X-ray; the third by digitalization.

\section{BODY-LANDSCAPE}

In the first moment, the artist and the anatomist portray the naked body in its surface, as a coherent whole. Science gives already attention to the interior body, the organs, the muscles, the vessels, the bones. But what matters is always the coherent amount of the elements, each one located in its own position, in its own function, in its dignity as a part of a never forgotten whole.

\section{BODY-FRAGMENT}

In the second moment, the camera and the X-ray capture analogical images which have a huge impact on medical knowledge and on the pictorial representation of the body. In Science, images are crossed by two fundamental movements: the shift from the naked eye drawing to an instrumentally mediated vision, the gradual deepening of the scale, into the inside, micro level structures. In the visual Arts, photography has also a remarkable impact. Free from the classical tasks of representation, works of art are invaded by other dimensions of the body put forward by the new $20^{\text {th }}$ century artistic approaches (fauvism, cubism, impressionism, expressionism, etc.). Both Science and Art enter the logic of fragmentation. And, even if the fragment is not a part, but is by itself a unity of sense, both Science and Art undertake the deconstruction of the body.

\section{BODY-ALGORITHM}

In the third moment, body images are now digital, that is, not graphically captured but computationally produced by mathematical algorithms (e.g. sound waves in the ultrasonography or the relaxation time of hydrogen nuclei in magnetic resonance). Biomedical sciences base their research programs on images acquired by different technologies (CT, MRI, PET, electron microscope, etc.). In Art, we witnessed again a proliferation of tendencies, such as the increasing use of time/movement (video-art) or the artistic exploitation of biological and biotechnological tools (bio-art). Further, body offers itself as surface of inscription for artistic activity. Body-art is gaining a new dimension by the use of robotics and prostheses that changes, modify and amplify body functions and his expression. That is to say, deeply contaminated by digital technologies, Art takes the body has the place where the limits of the body are experienced and thought out. 

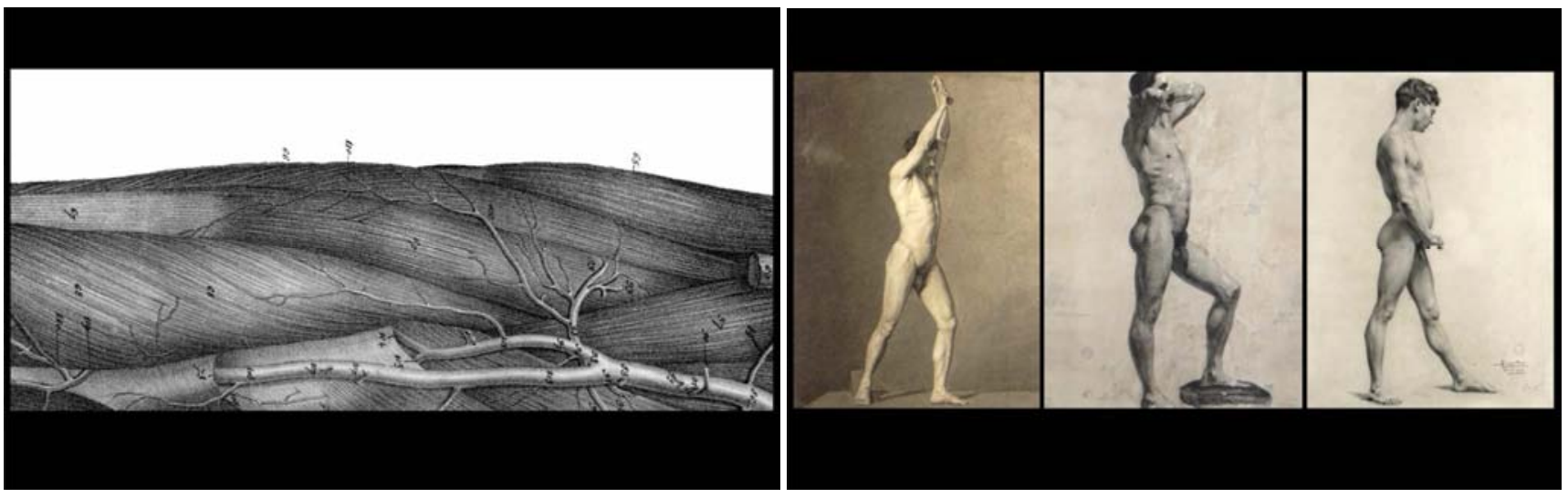

Figure 1: Body-landscape
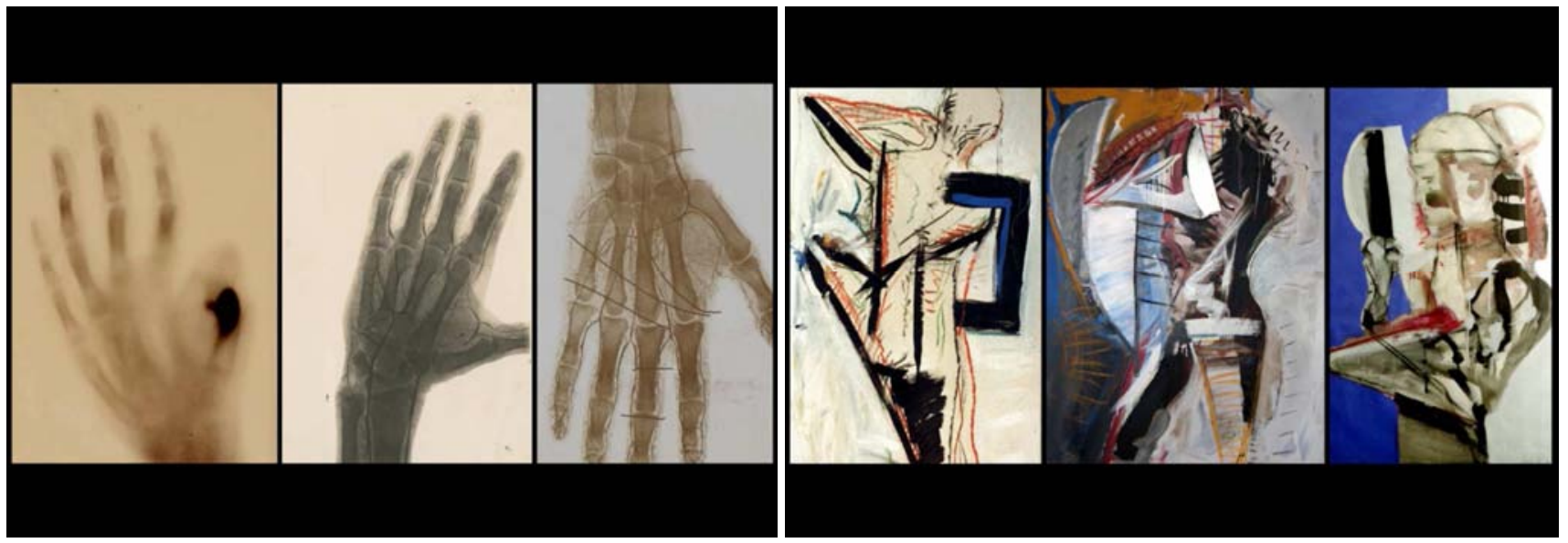

Figure 2: Body-fragment
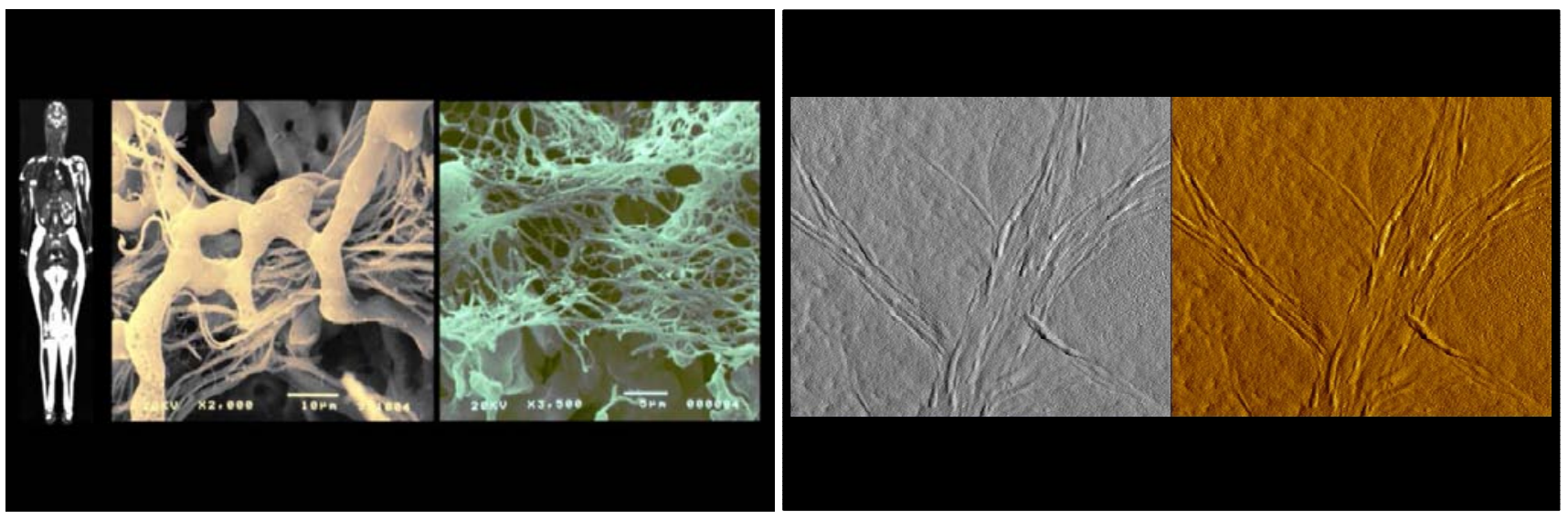

Figure 3: Body-algorithm 